\title{
Palm oil analysis in adulterated sesame oil using chromatography and FTIR spectroscopy
}

\begin{abstract}
This study highlights the application of two analytical techniques, namely GC-FID and FTIR spectroscopy, for analysis of refined-bleached-deodorized palm oil (RBD-PO) in adulterated sesame oil ( $\mathrm{SeO}$ ). Using GC-FID, the profiles of fatty acids were used for the evaluation of $\mathrm{SeO}$ adulteration. The increased concentrations of palmitic and oleic acids together with the decreased levels of stearic, linoleic, and linolenic acids with the increasing contents of RBD$\mathrm{PO}$ in $\mathrm{SeO}$ can be used for monitoring the presence of RBD-PO in SeO. Meanwhile, FTIR spectroscopy combined with multivariate calibration of partial least square (PLS) has been successfully developed for the detection and quantification of RBD-PO in SeO using the combined frequencies of $3040-2995,1660-1654$, and $1150-1050 \mathrm{~cm}-1$. The values of coefficient of determination (R2) for the relationship between actual versus FTIR-calculated values of RBD-PO in $\mathrm{SeO}$ and root mean square error of calibration (RMSEC) obtained are 0.997 and $1.32 \% \mathrm{v} / \mathrm{v}$, respectively. In addition, using three factors, the root mean square error of prediction (RMSEP) value obtained using the developed PLS calibration model is relatively low, i.e., $1.83 \%$ v/v. Practical Application: The adulteration practice is commonly encountered in fats and oils industry. It involves the replacement of high value edible oils such as sesame oil with the lower ones like palm oil. Gas chromatography and FTIR spectroscopy can be used as reliable and accurate analytical techniques for detection and quantification of palm oil in sesame oil.
\end{abstract}

Keyword: Adulteration; FTIR spectroscopy; Gas chromatography; RBD-palm oil; Sesame oil. 